\title{
Angiomiofibroblastoma como causa de hemorragia transvaginal
}

\author{
Angiomyofibroblastoma as a cause of transvaginal bleeding \\ Francisco Javier Ochoa Carrillo, * José Fernando Hernández Amador, ${ }^{\ddagger}$ \\ Tania Karina Cázares Robles, ${ }^{\ddagger}$ Adriana Cecilia Martínez López ${ }^{\S}$
}

\section{Resumen}

El angiomiofibroblastoma es una entidad nosológica con una incidencia baja y forma parte de los tumores estromales del tracto genital femenino; su presentación más común ocurre en la vulva. Posee un pronóstico favorable y representa un reto al realizar su diagnóstico diferencial, pues, aunado a la falta de consenso de criterios, hay una variedad de datos histopatológicos y marcadores inmunohistoquímicos. Se presenta el caso de una paciente postmenopáusica, quirúrgica que acudió al Servicio de Urgencias por sangrado incoercible postcoital e inestabilidad hemodinámica; se le realizó una resonancia magnética que evidenció un tumor en cúpula vaginal hiperintensa en T2, con posterior tumorectomía y evolución favorable. La localización vaginal del angiomiofibroblastoma es inusual, pues sólo hay siete casos reportados hasta 2004, según la revisión de Kaberi.

Palabras clave: Angiomiofibroblastoma vaginal, sangrado transvaginal, tumorectomía, tumores del estroma genital femenino.

\section{INTRODUCCIÓN}

El angiomiofibroblastoma (AMFB) fue descrito, por primera vez en 1992, por Fletcher; éste forma parte de los tumores estromales del tracto genital femenino, los cuales casi en su totalidad son benignos. Se presentan en mujeres entorno a los 46 años y además tienen una relación de 20:1 respecto de los hombres. Normalmente se ubican desde el endocérvix hasta la vulva. ${ }^{1,2}$

Histológicamente, estos tumores presentan bordes delimitados, pero sin cápsula, vasos de pared frágil y células

\section{Abstract}

The angiomyofibroblastoma is a low incidence entity that belongs to the stromal tumours of the female reproductive system, the most frequent location is the vulvar one. Even when it has a favorable prognosis, it represents a diagnostic challenge due to the lack of consensus criteria and the variety of histological data and immunohistochemical markers. We report the case of a surgical postmenopausal patient attending emergency room for postcoital bleeding and hemodynamic instability, with an evident hyperintense vaginal cuff tumor on the T2 magnetic resonance. Being treated with surgical resection, with adequate clinical evolution. Vaginal localization of angiomyofibroblastoma is unusual, with just 7 cases reported till 2004 by Kaberi.

Keywords: Vaginal angiomyofibroblastoma, transvaginal bleeding, tumorectomy, female genital tract stromal tumors.

\footnotetext{
* Consejo Mexicano de Oncología, Grupo Oncológico del Hospital Ángeles Pedregal. Ciudad de México, México.

* Médico Residente en Cirugía General. Facultad Mexicana de Medicina de la Universidad La Salle. Hospital Ángeles Pedregal. Ciudad de México, México.

$\S$ Médico pasante de Servicio Social, Universidad Popular Autónoma del Estado de Puebla. Puebla, Puebla.
}

fusiformes alrededor de los vasos, con matriz colagenosa edematosa con zonas de hipercelularidad, apariencia epitelioide que está asociada con núcleos periféricos, mitosis poco comunes, infiltración de tejido adiposo, cortes positivos a desmina y variabilidad a actina. ${ }^{3,4}$ En cuanto a su respuesta hormonal, se concluye que el angiomiofibroblastoma corresponde al tejido de origen. ${ }^{4}$

La ubicación más frecuente se encuentra en la vulva, pero es muy rara en la vagina, en las trompas de Falopio, perineo y en la uretra; en hombres se ubica en el escroto y en el cordón espermático. ${ }^{5}$ Sobre la presentación vaginal,
Correspondencia:

Dr. José Fernando Hernández Amador

Correo electrónico: grabberstang2@gmail.com

Aceptado: 26-09-2019.

www.medigraphic.com/actamedica 
en la literatura se reportan ocho casos: uno en Italia, en 2012 y, en una revisión de la literatura, siete casos desde la década de los sesenta hasta el año 2004.4-8

\section{CASO CLÍNICO}

Se presenta el caso de una mujer de 56 años con antecedente de duplicación ureteral, menarca a los 13 años con histerectomía total abdominal a los 33 años por miomatosis uterina y nuligesta; presenta ooforectomía bilateral por teratoma y su última citología vaginal fue normal. Su terapia de reemplazo hormonal se efectuó con estradiol $50 \mu \mathrm{g}$ dos veces por semana.

Inició seis meses previos con sangrado transvaginal postcoital, el cual era escaso, pero con remisiones parciales. En 10 ocasiones presentó sangrado espontáneo abundante con coágulos grandes, de coloración rojo brillante, el cual se asoció con dolor pélvico intenso e hipotensión que respondió a soluciones cristaloides.

Se le realizó una resonancia magnética (RM) con medio de contraste, la cual mostró una imagen ovoidea de bordes regulares, que midió $5.6 \times 3.6 \mathrm{~cm}$ en la cúpula vaginal (Figura 1). Sus estudios de laboratorio y marcadores tumorales estaban dentro de los parámetros normales. En la laparotomía exploradora se encontró un tumor de $7 \times 6 \mathrm{~cm}$, encapsulado, blanco nacarado y pediculado, que es dependiente de la cúpula vaginal pero que no invade los tejidos adyacentes (Figura 2).

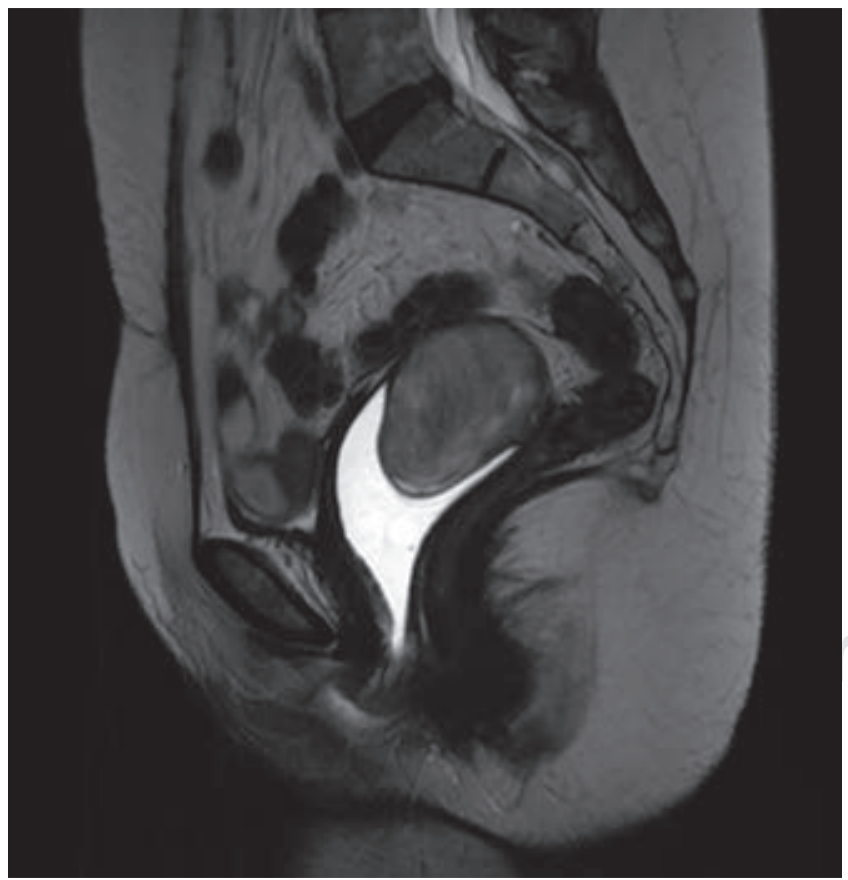

Figura 1: Resonancia magnética (RM) de T2, donde se muestra un corte sagital con gel en conducto vaginal.

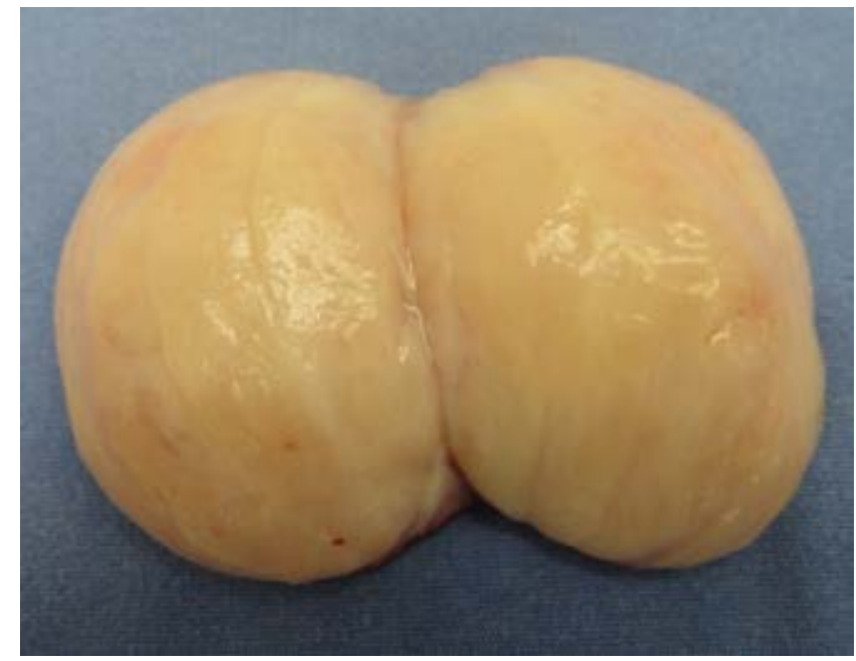

Figura 2: Pieza macroscópica de tumor de cúpula vaginal.

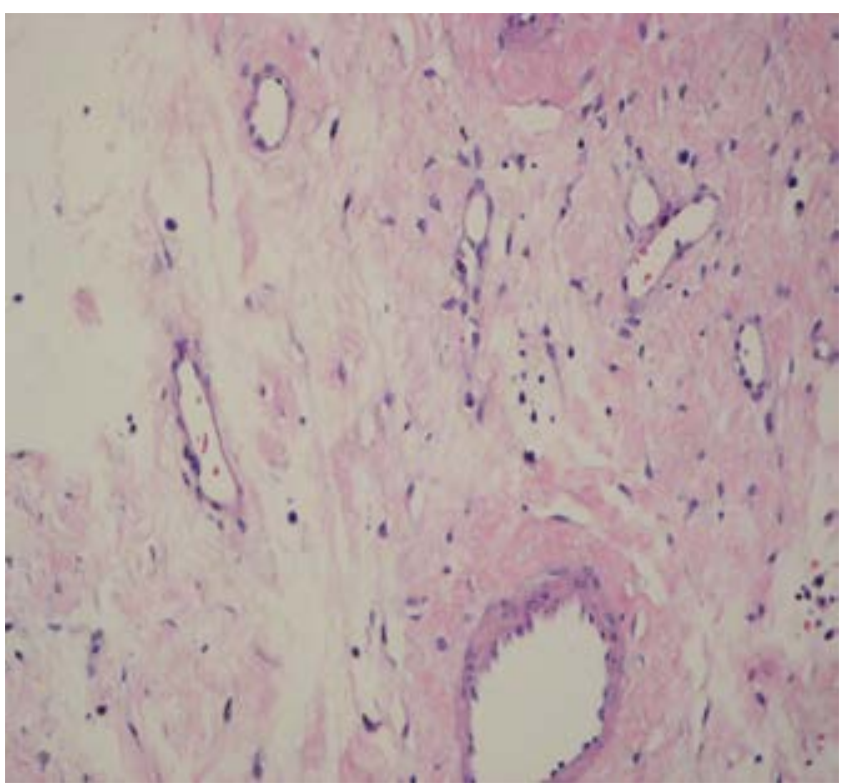

Figura 3: Imagen histológica con zonas hipocelulares que alternan con zonas hipercelulares en fibras de colágena.

La paciente cursó con una evolución favorable, sin presentar complicaciones postoperatorias. El estudio histopatológico transoperatorio reportó un tumor de origen estromal negativo para malignidad. El reporte definitivo identificó: CD34, desmina, receptores de estrógenos y receptores de progesterona positivos con CD10, actina de músculo liso, h-Caldesmon negativos, y $\mathrm{Ki}-67<5 \%$ compatible con angiomiofibroblastoma (Figura 3). 


\section{DISCUSIÓN}

Clínicamente, estos tumores se pueden presentar con dolor generalizado, sangrado postcoital y espontáneo, y sensación de pesantez; por ello, la revisión es oportuna en busca de fístulas hacia la vejiga o el recto. ${ }^{4,5}$ Genéticamente son diferenciables de angiofibroma y miofibroblastoma, por no encontrarse positivos a la mutación FoxO1 (deleción 13q14). ${ }^{6}$

Radiológicamente, no hay un estudio de elección, pero muestran sólo intensidad heterogénea en T2 y con reforzamiento rápido no homogéneo con gadolinio. ${ }^{7}$

Histopatológicamente, los diagnósticos diferenciales principales son angiomixoma profunda (agresivo), pólipo fibroepitelial, angiofibroma celular y miofibroblastoma extramamario. ${ }^{3,8}$

Su tratamiento no está bien definido, actualmente consiste en tumorectomías con escisión local y márgenes limpios con estudios transoperatorios, además de su posterior seguimiento. ${ }^{1,8}$

El angiomiofibroblastoma es una entidad rara que tiene una etiología multifactorial, aunque posee un pronóstico benigno y una mortalidad casi nula; cursa con una morbilidad variable, que es dependiente de su ubicación. En la literatura mundial, sólo se han reportado 15 casos de su ubicación vaginal, sin embargo, cada vez más, se identifican y dan a conocer más casos que permitan realizar una intervención temprana y señalar las diferencias con otras etiologías similares. ${ }^{9,10}$

\section{REFERENCIAS}

1. Fletcher CD, Tsang WY, Fisher C, Lee KC, Chan JK. Angiomyofibroblastoma of the vulva. A benign neoplasm distinct from aggressive angiomyxoma. Am J Surg Patho. 1992; 16: 373-382.

2. Horvai A, Link T. "Angiomyofibroblastoma". In: Bone and soft tissue pathology: high-yield pathology. E.U.A.: Saunders, Elsevier; 2012. pp. 315-316.

3. Nucci M. Mesenchymal lesions of the lower genital tract. Surg Pathol Clin. 2009; 2 (4): 603-623.

4. McCluggage W. Immunohistochemical markers of value in the diagnosis of mesenchymal lesions of the female genital tract. Surg Pathol Clin. 2009; 2 (4): 785-811.

5. McCluggage W, White RG. Angiomyofibroblastoma of the vagina. J Clin Pathol. 2000; 53: 803-806.

6. Banerjee K, Datta Gupta S, Mathur SR. Vaginal angiomyofibroblastoma. Arch Gynecol Obstet. 2004; 270 (2): 124-125.

7. Magro G, Righi A, Caltabiano R, Casorzo L, Michal M. Vulvovaginal angiomyofibroblastomas: morphologic, immunohistochemical, and fluorescence in situ hybridization analysis for deletion of $13 q 14$ region. Hum Pathol. 2014; 45 (8): 1647-1655.

8. Geng J, Hu S, Wang F. Large paravaginal angiomyofibroblastoma: magnetic resonance imaging findings. Jpn J Radiol. 2011; 29: 152-155.

9. Lee H, Jeng C, Ko M, Huang S, Chen S. Vulvar Angiomyofibroblastoma. J Obstet Gynecol. 2008; 47 (2): 220-221.

10. Magro G, Caltabiano R, Kacerovská D, Vecchio GM, Kazakov D, Michal M. Vulvovaginal myofibroblastoma: expanding the morphological and immunohistochemical spectrum. A clinicopathologic study of 10 cases. Hum Pathol. 2012; 43 (2): 243-253. 